\title{
Heart transplant pathology: the British experience
}

\author{
ARIELA POMERANCE, PGI STOVIN \\ From the Departments of Histopathology, Harefield and Mount Vernon Group Laboratories, Middlesex, and \\ Papworth Hospital, Cambridge
}

SUMMARY An account of human heart transplantation as seen by the histopathologists involved at the two UK transplant centres is presented. Between January 1979 and July 1984179 patients received 186 hearts and 124 are still alive up to four years after operation. Cyclosporin A based immunosuppression has been used in the last 120 patients. Four patients developed neoplastic lesions. The commonest reason for transplantation was ischaemic heart disease $(63 \%)$, followed by congestive cardiomyopathy $(35 \%)$. The seven retransplants were for acute or chronic rejection. The monitoring of rejection by endomyocardial biopsies is described, and the causes of death and necropsy findings are presented.

Since Barnard' performed the first human heart transplant in 1967 , over 1000 patients world wide have received new hearts. Most transplants have been orthotopic, in which the old heart is removed leaving the posterior walls of the atria and the donor heart is sutured to the atrial remnants and great vessels. Initial survival rates were poor, and it was not until late 1979 , after improved immunosuppressive agents and safe endomyocardial biopsy had become available, that the UK transplant programme was reactivated.

The two UK cardiac transplant centres are at Papworth and Harefield hospitals, where between January 1979 and July 1984186 transplants were performed on 179 patients, including 20 heterotopic transplants in the 110 patients operated on at Harefield. In this operation, as pioneered by Barnard and his team ${ }^{2}$ the hearts are joined by their atria and great vessels so that the new heart works in parallel with the old. The seven retransplants, done between 10 days and about three years after the first operation, were performed for acute or chronic rejection. Both centres initially used antithymocyte globulin, prednisolone, and azathioprine for immunosuppression. Both now use cyclosporin $\mathrm{A}$, which appears to be a selective inhibitor of T cells. ${ }^{3}$ Both rely extensively on endomyocardial biopsy for monitoring rejection.

Interest in cardiac transplantation is increasing and is encouraged by the present results. A hundred and twenty four of our 174 patients who survived the operation are still alive, and these include 13

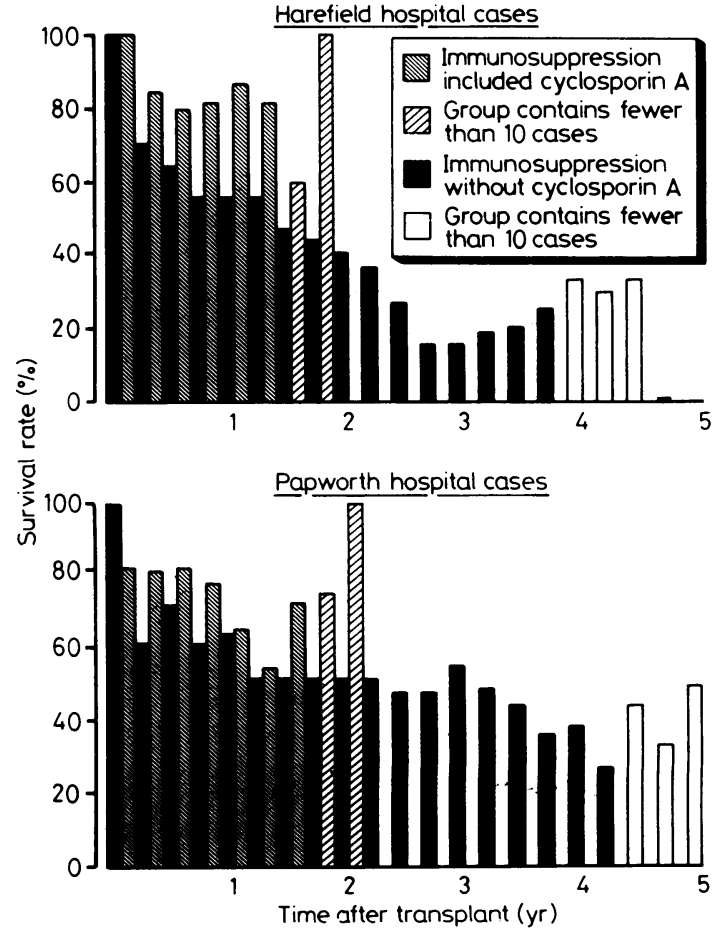

Fig. 1 Survival rates for heart transplant recipients at Papworth and Harefield hospitals.

patients operated on more than three years ago (Fig. 1). The results have been greatly improved by cyclosporin $\mathrm{A}$, which was introduced in the first quarter of 1982 in Papworth and the last quarter in 
Harefield. This drug has reduced the frequency and severity of both rejection and infective episodes, and the one year actuarial survival of patients treated with cyclosporin $A$ is now about $75 \%$. While few pathologists are likely to be concerned with monitoring cardiac rejection the likelihood of performing necropsies on these patients is increasing. We therefore present our experiences to date and describe what involvement in a heart transplant programme entails for the histopathologist.

\section{The recipient heart}

The causes of the recipients' cardiac failure are shown in Table 1. The pathology of these conditions is amply described in the published work and we do not propose to consider it here beyond pointing out the obvious opportunity to examine substantial amounts of fresh tissue from severely diseased hearts. This examination will make considerable demands on the time of histopathologist and laboratory staff, however, and almost invariably occurs out of laboratory hours.

\section{Control of immunosuppression}

The transplant patient needs to maintain a stable balance between a level of immunosuppression sufficient to prevent rejection but one low enough to avoid an unacceptable risk of opportunistic infection.

For patients on conventional immunosuppression with azathioprine and steroids plus antithymocyte globulin, measurement of peripheral blood $\mathrm{T}$ cell count is of some use in monitoring rejection ${ }^{45}$ but this is of no value in patients treated with cyclosporin $A .^{67}$ Identification of $\mathrm{T}$ cell subsets have not so far proved helpful in monitoring immunotherapy or detecting rejection episodes, ${ }^{8}$ although they may be of use in detecting infection. $\mathrm{O}^{\prime}$ Toole $^{9}$ has reported that the suppressor to helper $\mathrm{T}$ cell ratio in peripheral blood is reversed in infection but not in rejection.

In cardiac transplantation the mainstay of detection and control of rejection is transvenous right ventricular biopsy, as described by Caves et al..$^{10}$ This is a relatively safe procedure. Postoperative

Table 1 Aetiology of recipient's heart disease

\begin{tabular}{lc}
\hline & No of patients \\
\hline Ischaemic & 113 \\
Cardiomyopathy (COCM) & 62 \\
Valvular heart disease & 5 \\
Congenital heart disease & 8 \\
Tumour (low grade neurofibrosarcoma) & 1 \\
\hline
\end{tabular}

adhesions prevent tamponade if the bioptome penetrates the ventricular wall, and the most troublesome of the infrequent complications is pneumothorax.

Biopsies are done at roughly weekly intervals during the first month after operation and then every two weeks. After discharge biopsies usually continue at 3, 4, and 6 months postransplant and then annually. Additional biopsies are done if rejection is suspected because of reduced electrocardiogram voltages, fever, rhythm changes, heart failure, or weight gain. At Papworth, with five new cases every two months and 52 surviving patients, this entails about nine cardiac biopsies a week. At Harefield, with four to six new patients a month and 75 surviving patients, 9 to 24 such biopsies are performed each week.

The introduction of cyclosporin $A$ based immunosuppression into the UK heart transplant programmes has influenced the histopathology as well as prognosis. With this drug, which appears to have a selective effect on T cells, ${ }^{4}$ rejection develops and resolves more slowly, the risk of intercurrent infection is less, but nephrotoxicity is an important postoperative problem. The classic changes of rejection still occur, although less often, but additional features are added to those which may be noted in the endomyocardial biopsy.

\section{Technical aspects}

There is some variation in the techniques used in our laboratories. Rapid processing must be available because of the need for urgent treatment in severe rejection, although the introduction of cyclosporin $A$ has reduced the frequency of urgent biopsies. Both centres initially tried conventional frozen sections but found them unsatisfactory except in severe rejection. Papworth now uses a $2 \mathrm{~h}$ paraffin processing cycle (Autotechnicon Ultra II) and sections are ready for reading $4 \mathrm{~h}$ after receipt. The Mount Vernon/Harefield laboratory processes urgent cases manually, also providing a report within $4 \mathrm{~h}$, while non-urgent cases are processed overnight with the routine non-cardiac biopsies.

The microscopical changes of rejection are patchy and so normally at least three samples of myocardium are taken to reduce the chance of missing acute rejection to $5 \% .^{11}$ Biopsies are fixed in $10 \%$ neutral buffered formalin, which shows interstitial oedema better than the traditional rapid fixatives such as Carnoy, which is used for Harefield biopsies. All the pieces are embedded in a single block with no attempt at orientation. This is serially sectioned, providing 10-12 slides each with a ribbon of four to eight sections. Representative levels are stained with 

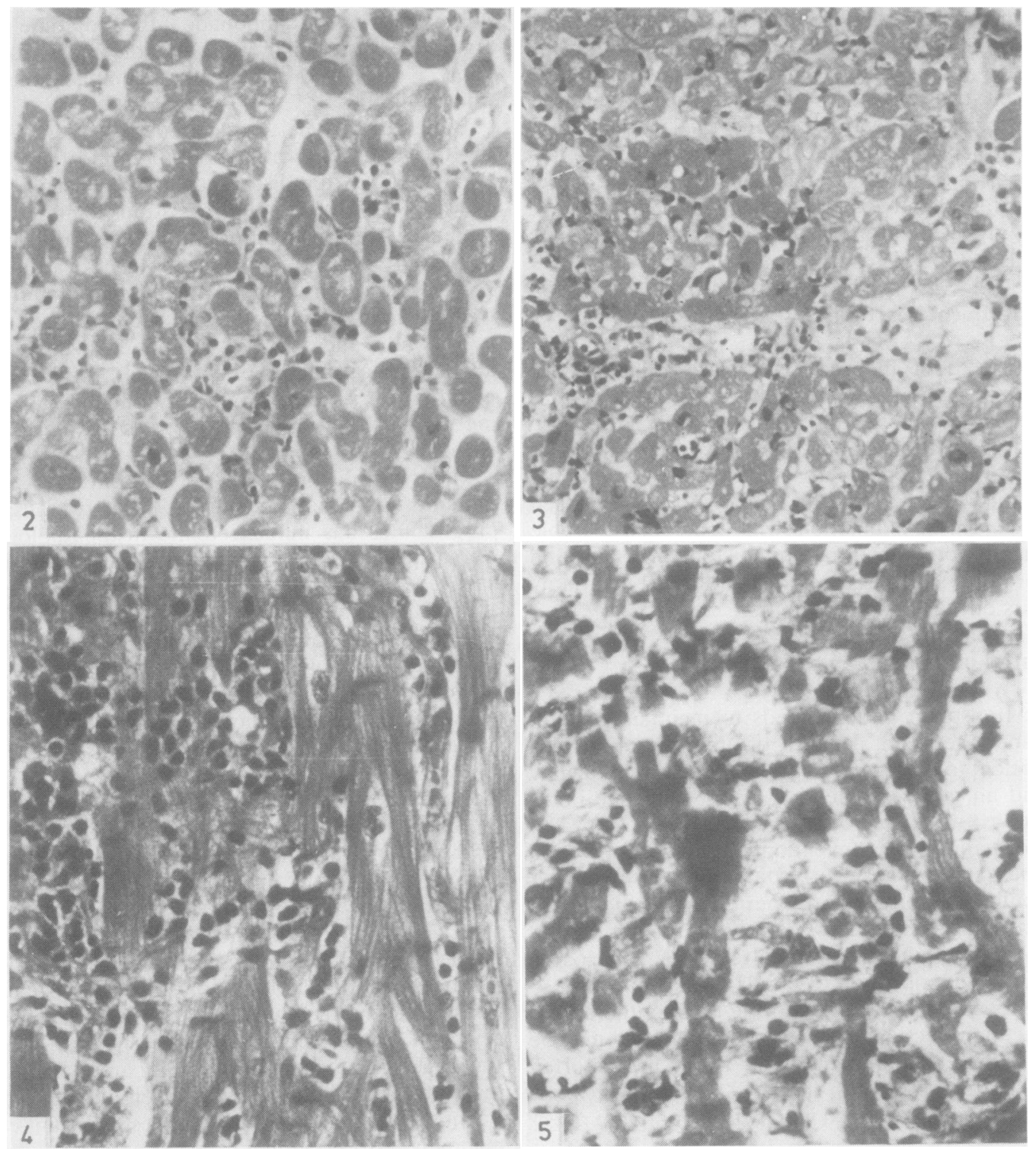

Fig. 2 Mild rejection. Small collections of interstitial lymphocytes are present. Haematoxylin, phloxin, saffron (HPS).

Fig. 3 Moderate rejection. More frequent and more intense cellular infiltration with commencing damage to myocytes. HPS.

Fig. 4 Moderate rejection, of more intense degree than on Fig. 2. HPS.

Fig. 5 Severe rejection showing dense cellular infiltration which includes neutrophils. HPS.

haematoxylin, phloxin, and saffron (Papworth) or haematoxylin and eosin (Harefield) and intervening levels stained with methyl green pyronine and Masson trichrome, leaving the remainder as spares. The haematoxylin stained slides show the extent, dis- tribution, and composition of the cellular infiltrate and also show the myofibre morphology. Methyl green pyronine staining shows the degree of pyroninophilia and the proportion of mononuclear cells with RNA activity and is therefore a good indi- 
Table 2 Diagnostic grades* of acute cell mediated cardiac rejection as seen in endomyocardial biopsies

\begin{tabular}{|c|c|}
\hline Grade & Microscopical features \\
\hline Minimal & $\begin{array}{l}\text { A few pyroninophilic cells, either endocardial or } \\
\text { interstitial, present in only a few adjacent sections; } \\
\text { the biopsy was otherwise normal. }\end{array}$ \\
\hline Mild & $\begin{array}{l}\text { Scattered foci of interstitial, perivascular, or } \\
\text { endocardial pyroninophilic mononuclear cells in } \\
\text { many sections. Interstitial oedema. No damage } \\
\text { to myocytes. }\end{array}$ \\
\hline Moderate & $\begin{array}{l}\text { More intense infiltration than in mild, with damage } \\
\text { to single or small groups of myocytes. }\end{array}$ \\
\hline Severe & $\begin{array}{l}\text { Increased cellular infiltration with neutrophils or } \\
\text { haemorrhage or both. Larger groups of myocytes } \\
\text { showing damage. }\end{array}$ \\
\hline Resolving & $\begin{array}{l}\text { Remaining mononuclear cells are smaller and } \\
\text { non-pyroninophilic. Decreasing cellular infiltrate } \\
\text { may be accompanied by or replaced by active } \\
\text { fibrosis, scarring, and lipochrome pigment in } \\
\text { macrophages. }\end{array}$ \\
\hline
\end{tabular}

${ }^{*}$ Based on Billingham's criteria. ${ }^{12}$

cator of immunological activity in the cellular infiltrate. The Masson stain is mainly of value for confirming fibrosis. Myocyte degeneration is usually equally clear in the haematoxylin stained slides.

\section{Grading of rejection}

The severity of rejection is graded none to severe, based on the features described by Billingham ${ }^{12}$ (Table 2; Figs. 2-5). The grade reported and acted on is the most severe change seen in any one of the biopsy pieces. Although distinctive features are defined for each grade, it is not unusual to find different grades in two or more fragments in the same block. The process of rejection is an evolving/ regression continuum, starting with small pericapillary groups of mononuclear cells widely but fairly uniformly scattered throughout the myocardium. As the rejection episode intensifies these groups enlarge and new ones develop, and not all are at the same stage of evolution at the time of biopsy. If rejection becomes severe all the biopsy fragments are more uniformly affected.

Although most acute rejection episodes occur in the first six months after transplantation, they may continue to occur after this but with decreasing frequency. Montiès et $\mathbf{l}^{13}$ reported an acute rejection episode 67 months after operation. It is reassuring that normal myocardium has been seen in endomyocardial biopsies eight years after transplantation $^{14}$ and the patient of Montiès et al $^{13}$ survived at least 13 years despite showing evidence of chronic rejection.

\section{Non-rejection pathology}

The most common finding which may confuse the histological assessment of rejection is inclusion of previous biopsy sites (Figs. 6-8). This is more frequent in some patients than others, probably due to anatomical configuration. Pathological changes at biopsy sites range from loss of endothelium and fibrin deposition (Figs. 6 and 7) through inflammatory cell infiltration, fibroblastic proliferation (Figs. 7 and 8), to fibrous scarring. Endocardial inflammatory response without myocardial evidence of rejection is clearly due to biopsy trauma, but subendocardial inflammatory cell foci may be difficult to distinguish from the cellular infiltrate of rejection. Serial sections may establish continuity with obvious biopsy site changes and the methyl green pyronine stain often shows numerous strongly pyroninophic plasma cells which would be absent in a rejection response. Myocytes running at an angle to the endocardium (Fig. 8) also indicate a biopsy site. Subendocardial myocytes in the normal heart appear parallel to the endocardium and a nonparallel arrangement is due to endothelialisation of the deeper muscle cells exposed after biopsy.

A more difficult problem may be distinguishing the cellular infiltration associated with viral or parasitic infections from that of rejection since these infections may produce multifocal lymphocytic infiltrates with necrosis of occasional myocytes, similar to the picture of moderate rejection. An associated fever and fall in circulating $T$ cell count suggests cytomegalovirus infection but there may be no changes in the early stages of most other infections. Stains which distinguish helper and suppressor $T$ cells were found helpful in identifying cytomegalovirus infection in renal transplants ${ }^{15}$ but have not been of value in the cardiac cases. ${ }^{68}$

Clearly, finding cytomegalovirus changes in nuclei or toxoplasma cysts in myofibres (Fig. 9) makes it most unlikely that the cellular response is due to rejection. Parasites were seen in biopsy material from three of the four patients with toxoplasma myocarditis in the Papworth series. These three patients were among the five recipients with negative toxoplasma antibodies who received hearts from donors with serological evidence of past infections. We have not yet seen toxoplasma myocarditis where both recipient and donor had serological evidence of past infection. A histological feature which has been noted in biopsies from toxoplasma myocarditis is the presence of small myocyte sized fibrotic areas, ${ }^{16}$ which may be the late result of previous myofibre necrosis produced by the parasite.

An occasional problem is interpretation of minor foci of myocyte damage or recent replacement fibrosis. Possible causes include: catecholamine induced damage to the donor heart before transplantation ${ }^{17}$ 18; $^{2}$ resolved rejection with either cytotoxic immune change affecting groups of myo- 

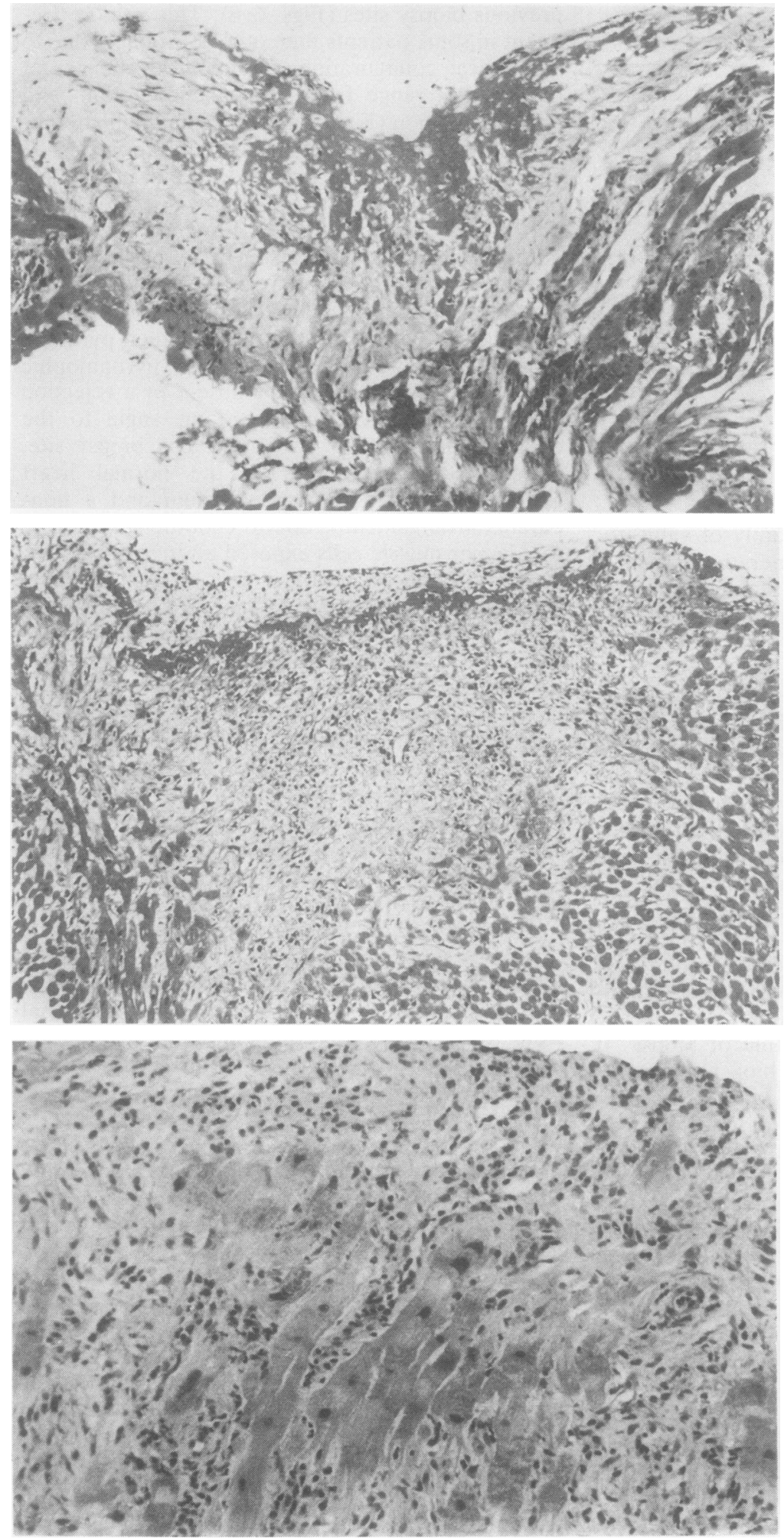

Fig. 6

Fig. 7

Figs. 6-8 Previous biopsy sites.

Fig. 6 shows a cup shaped

subendocardial defect lined by

fibrin and surrounded by fibrous tissue. Fig. 7 is a more recent cellular lesion incorporating a thin layer of fibrin. Fig. 8 shows myocytes extending perpendicularly to the endocardial surface, with cellular fibrous tissue. A little fibrin is present on the endocardial surface towards the left. Figs. 6 and 7 haematoxylin and eosin $(H \& E)$. Fig. 8 HPS.

Fig. 8 


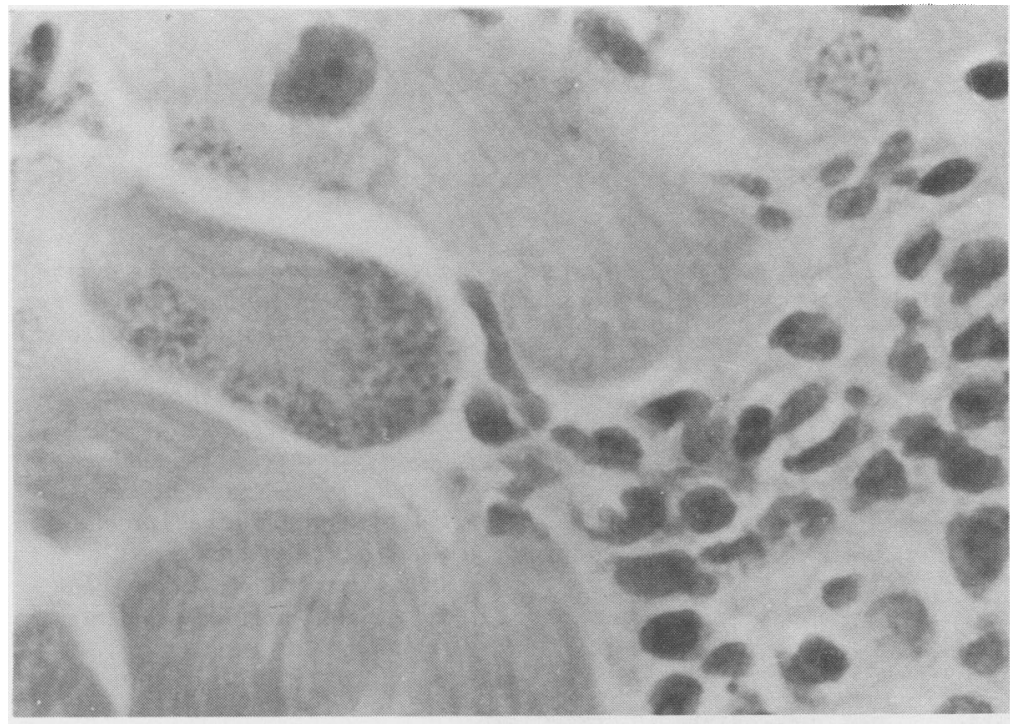

Fig. 9 Toxoplasma cysts with adjacent infiammatory reaction in a right ventricular endomyocardial biopsy. HPS. cytes or immune vaculitis affecting intramyocardial arterioles and producing small ischaemic lesions; damage to arterioles, up to about $100 \mu \mathrm{m}$, by previous biopsy. If none of these seems to apply then focal degeneration and scarring may indicate chronic rejection. This term refers to an accelerated form of vascular disease which affects predominantly the smaller epicardial coronary artery branches, $0.5-1 \mathrm{~mm}$ diameter. It has been seen as early as the ninth day after transplant ${ }^{19}$ but does not usually become important before the third month.

Occasionally, a deep biopsy includes epicardial fat, which almost always shows mild lymphocyte and plasma cell infiltration which does not correlate with the usual infiltrates of rejection. Sometimes frank fat necrosis has been seen in patients who had required active resuscitative measures.

Contraction bands are almost invariably present in the biopsies and their absence would suggest impaired myocardial function. In cross section they may appear as pale "degenerate" myocytes. This appearance musi be distinguished from vacuolation within only part of the cross section of a fibre. Vacuolation is also a common finding and suggests early ischaemic damage which appears reversible.

The cyclosporin effect is not strictly non-rejection pathology since it was not seen in the native hearts of our heterotopic transplant patients. Morphologically, there are two types of reaction. The cellular type (Figs. 10 and 11 ) is seen in the early postoperative period and has been most common in Harefield patients with blood cyclosporin concentrates greater than $400 \mathrm{ng} / \mathrm{ml}$. In Carnoy fixed biopsies it is characterised by a pronounced pyroninophilic interstitial oedema and diffuse cellular infiltrate with a low proportion of pyroninophilic cells. Dense endocardial mononuclear cell infiltration, is often a striking feature. Many of the cells stain for $\alpha_{1}$-antitrypsin and muramidase with immunoperoxidase stains, indicating a histiocytic origin. Myofibres may be interrupted by groups of large mononuclear cells, but myofibre damage appears much less than that which would be associated with a comparable density of cellular infiltration in patients treated by conventional (non-cyclosporin A) immunosuppression. Even our most alarming examples of this effect have resolved under treatment and progress to a diffuse fine interstitial fibrosis (Fig. 12). Unlike the Stanford cases, which all showed fibrosis, ${ }^{12}$ only the Harefield cases with previous cellular changes have so far shown this fibrous type of cyclosporin A effect; in the Papworth experience there has been no difference in incidence or severity of fibrosis in patients treated with cyclosporin $A$ and those receiving azathioprine and antithymocyte globulin.

\section{Postmortem pathology}

When a heart transplant recipient dies every effort is made to secure an early necropsy, and necropsies have been performed in all but one of our cases to date. If the postmortem examination was not actually carried out by one of us we have always been sent the relevant organs by the pathologist concerned. Table 3 summarises the findings.

In the intraoperative and immediate postoperative period death may be due to cardiac arrest during anaesthesia and before bypass is established; this 


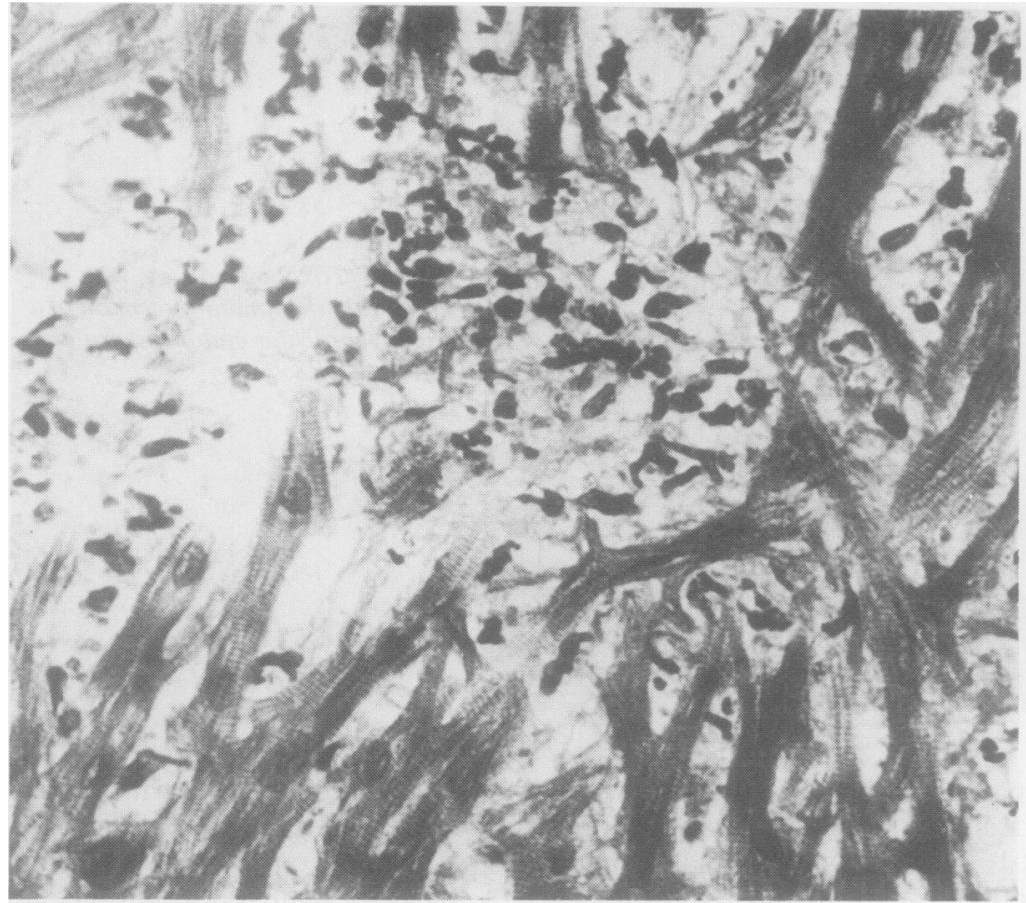

Fig. 10 Cyclosporin effect as seen in the earlier Harefield patients. The myocardium shows marked oedema with dense cellular interstitial infiltrates including numerous rather large mononuclear cells. The picture is like that of severe rejection except that pyroninophilic cells are not numerous and there is no significant myofibre damage. The patients had no clinical evidence of rejection.

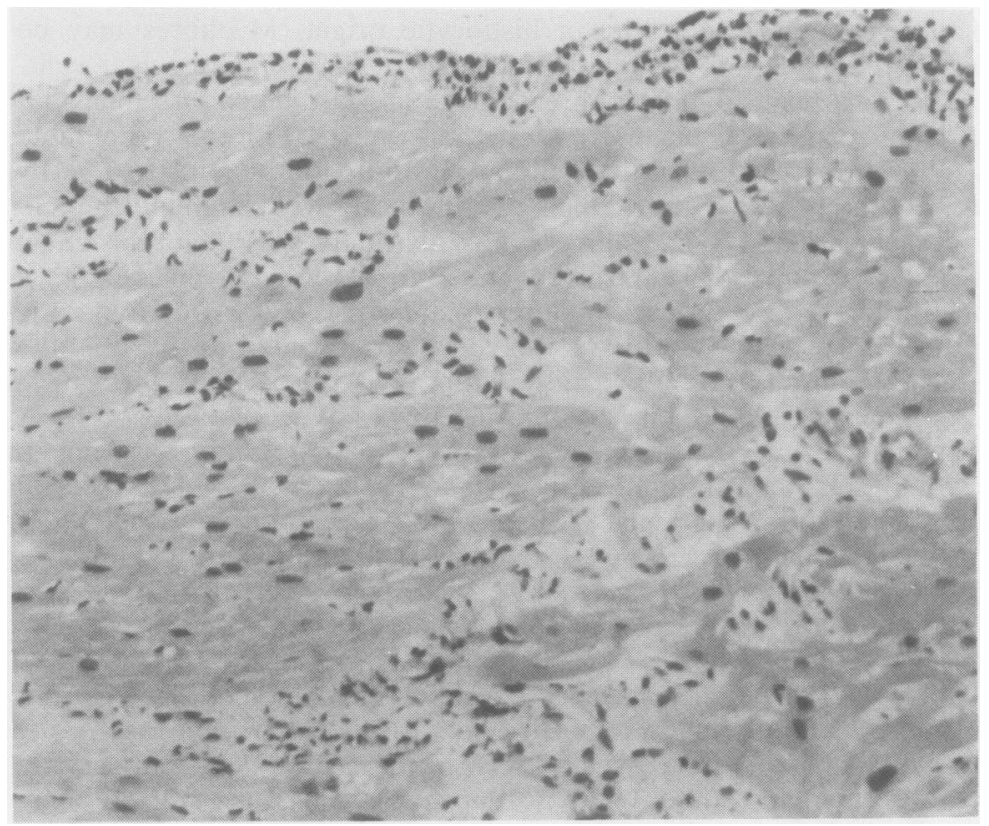

Fig. 11 Cyclosporin effect as seen in current Harefield patients. Interstitial oedema and cellular infiltration are less pronounced and endocardial cellular infiltration, mainly mononuclear, is prominent.

is a critical time for patients with end stage heart disease. Previous heart surgery may cause surgical hazards resulting in excessive bleeding. The donor heart may be unsuitable for reasons that were not evident before operation. We have had three cases of unsuspected donor heart disease: one from each centre with donor myocarditis (Fig. 13) and one with an anomalous coronary sinus drainage (Pap- 
Table 3 Summary of necropsy findings



$\mathrm{c}=$ patients receiving cyclosporin $\mathrm{A}$

$\mathrm{h}=$ heterotopic transplant.

$\mathrm{v}$ to $\mathrm{z}=$ the same patient.

$v$ to $y=$ had second transplants. The higher figure is the time at which the first heart was removed; the lower is survival time with the second heart.

worth). One donor heart (Harefield) proved inadequate to cope with a recipient's raised pulmonary vascular resistance.

Apart from one patient with unresponsive renal failure and one with carcinoma, later deaths have been due to infection or rejection. All but one of the infective deaths occurred in the first postoperative year. Table 3 and Figs. 14 and 15 list and illustrate some of the organisms found in our cases and papers by Jamieson $e t a^{20}$ and Williams $e t a^{21}$ list the organisms found at other centres. Pulmonary and septicaemic infections are the most common, followed by urinary tract and central nervous system infections, but no site is exempt. Our cases have included meningitis secondary to otitis media and fungal aortitis. Fortunately, many infections are not fatal. An aggressive approach to early diagnosis of febrile episodes and pulmonary radiological opacities allows effective treatment and the histopathologist may expect to receive lung biopsies (Figs. 14 and $15)$ as well as the endomyocardial biopsies.

Deaths due to rejection fell into two groups. Acute rejection, characterised by interstitial myocardial cellular infiltrates, was seen in the first two postoperative months (Figs. 2-5) while chronic rejection changes, affecting arteries and arterioles, (Figs. 16-18) were the dominant pathology after this period.

The gross pathology of the heart in acute rejection is distinctive, with a solid "beefy" consistency and fine haemorrhagic mottling of the endocardial surface. This contrasts with the recipient's uniformly pale atrial endocardium. The cut surface of both ventricles also shows fine purple and yellowish 




Fig. 12 Fine interstitial fibrosis in a patient who had previously shown changes like those illustrated in Fig. 10.

mottling.

Microscopically, the myocardium has usually shown the features of moderate or pronounced grades of rejection previously described. Vigorous antirejection treatment may reduce the intensity of the cellular infiltrate but cannot influence estab-

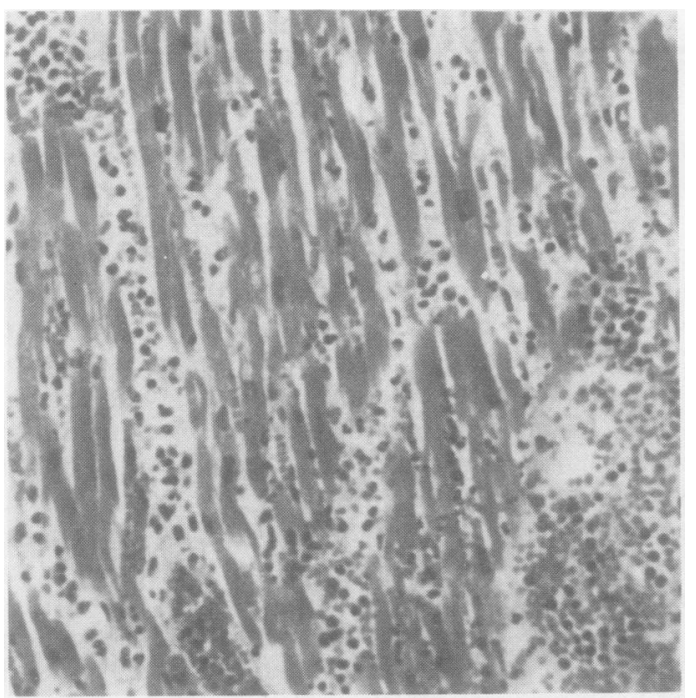

Fig. 13 Donor myocarditis. Section from the right ventricle of a donor heart which failed shortly after transplantation, showing marked interstitial inflammatory cell and erythrocyte infiltration. $H \& E$. lished myofibre damage. One of our patients died of paradoxical embolism during treatment of a moderately severe rejection episode and showed widespread myofibre degeneration with relatively little cellular infitration. Intramyocardial vascular lesions may be present. The cellular infiltration is not dense, as in polyarteritis nodosa, microaneurysms do not form, and affected vessels are therefore not visible to the naked eye. Valves, veins, and great vessels may be normal or show variable, usually minor, infiltration by mononuclear cells.

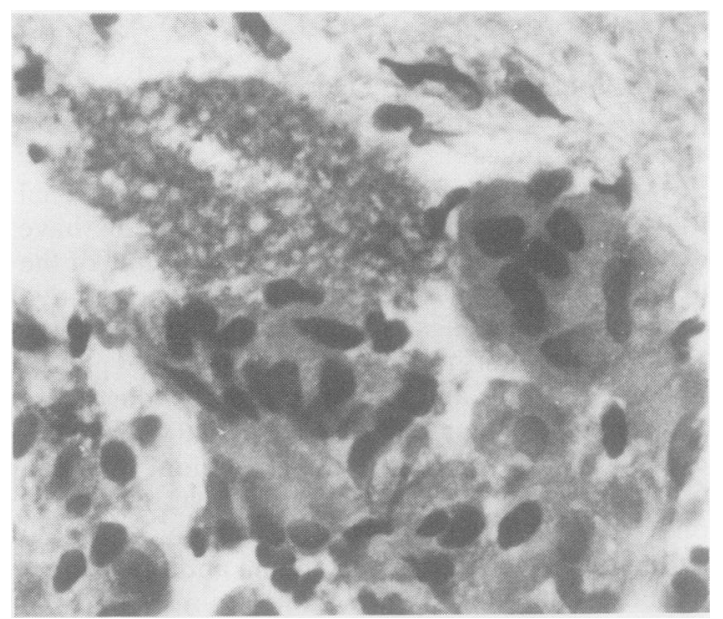

Fig. 14 Pneumocystis in a lung biopsy. HPS. 


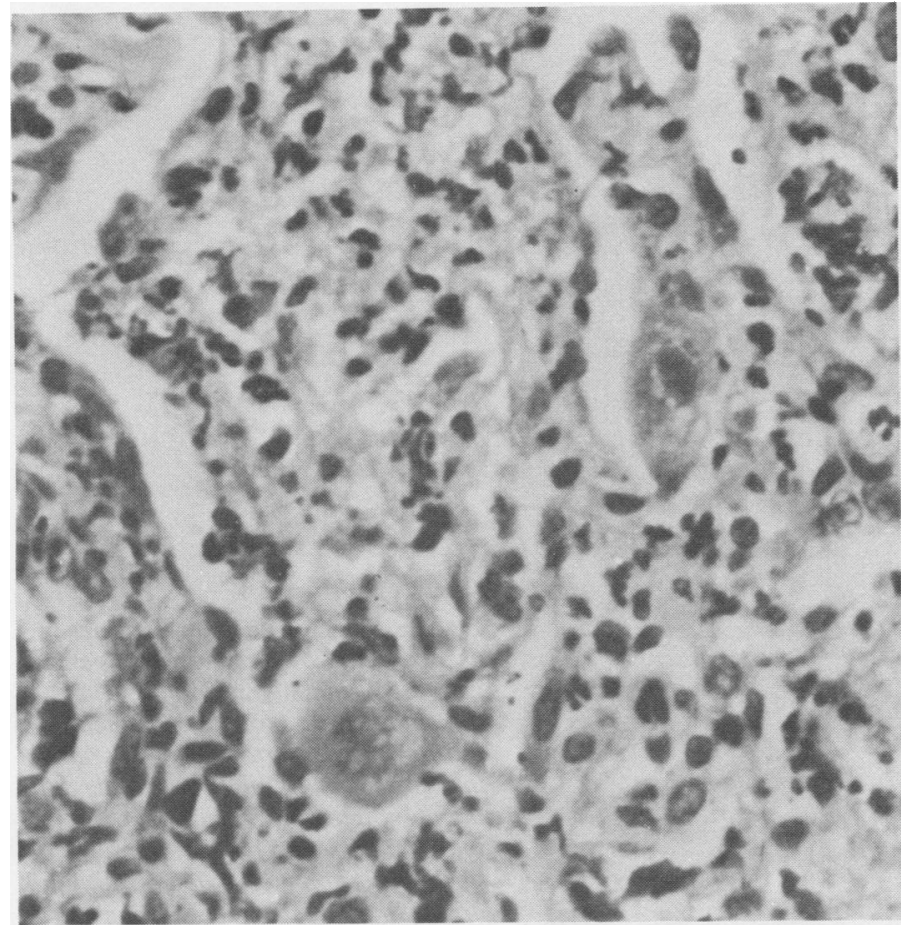

Fig. 15 Cytomegalovirus in a lung biopsy 135 days after transplantation. This patient is alive and well over four years after operation.

Chronic rejection primarily affects the coronary arteries and may be accompanied by changes of acute rejection in the myocardium. Macroscopically, the main vessels may appear slightly thickened, and soft eccentric yellow plaques have been seen in some of the Harefield cases (Figs. 16 and 17) although not, so far, in the Papworth series. In two of the four chronic rejection cases from Harefield bright yellow intramyocardial arteries stood out clearly on cutting the left ventricle (Fig. 16) and one showed mild localised aneurysmal dilatation of the anterior descending coronary artery. Thrombosis may occur as a result of poor run off due to narrowing of smaller artery branches, and in one of our cases fatal thrombotic coronary occlusion was associated with fibrinoid necrosis of main coronary arteries. This and another case with thrombus on focal atherosclerotic plaques in the right main coronary artery were the only cases to show frank infarction, although small subendocardial fibrous scars are not uncommon. Because the transplanted heart is denervated these patients do not have angina, and symptoms, if any, have been effort dyspnoea and those due to low cardiac output.

Microscopically (Figs. 17 and 18), the arteries show intimal thickening by cellular loose fibrous and fibroblastic tissue, which may completely occlude the lumen. The elastic laminae may be intact or fragmented, and the media may be infiltrated by mononuclear cells. Lipid plaques may be present between the fibrocellular intimal thickening and the internal elastic lamina, but whether these are related to pretransplant donor atheroma or to the "accelerated atheroma" of chronic rejection is speculative. Acute arteritis of small intramyocardial vessels has also been seen in our chronic rejection cases (Fig. 19).

\section{Heterotopic transplantation}

To date 20 patients in the Harefield series have undergone heterotopic transplants. Three have died, 20-46 days after operation. Initially, both hearts were biopsied, which allowed comparison of the effects of rejection and treatment in 11 pairs of biopsies from patients. Not unexpectedly, rejection was seen only in the donor heart. The recipient hearts were either unremarkable or showed features of their preoperative pathology. The most interesting finding was the difference between donor and recipient biopsies in patients being treated with cyclosporin $\mathrm{A}$. The pronounced interstitial oedema and cellular infiltration often found with high blood cyclosporin concentrations was seen only in the donor heart, thus confirming its essentially immune basis. Unlike Uys and Rose ${ }^{22}$ we have not found endomyocardial biopsies less likely to be satisfactory than in orthotopic hearts. 

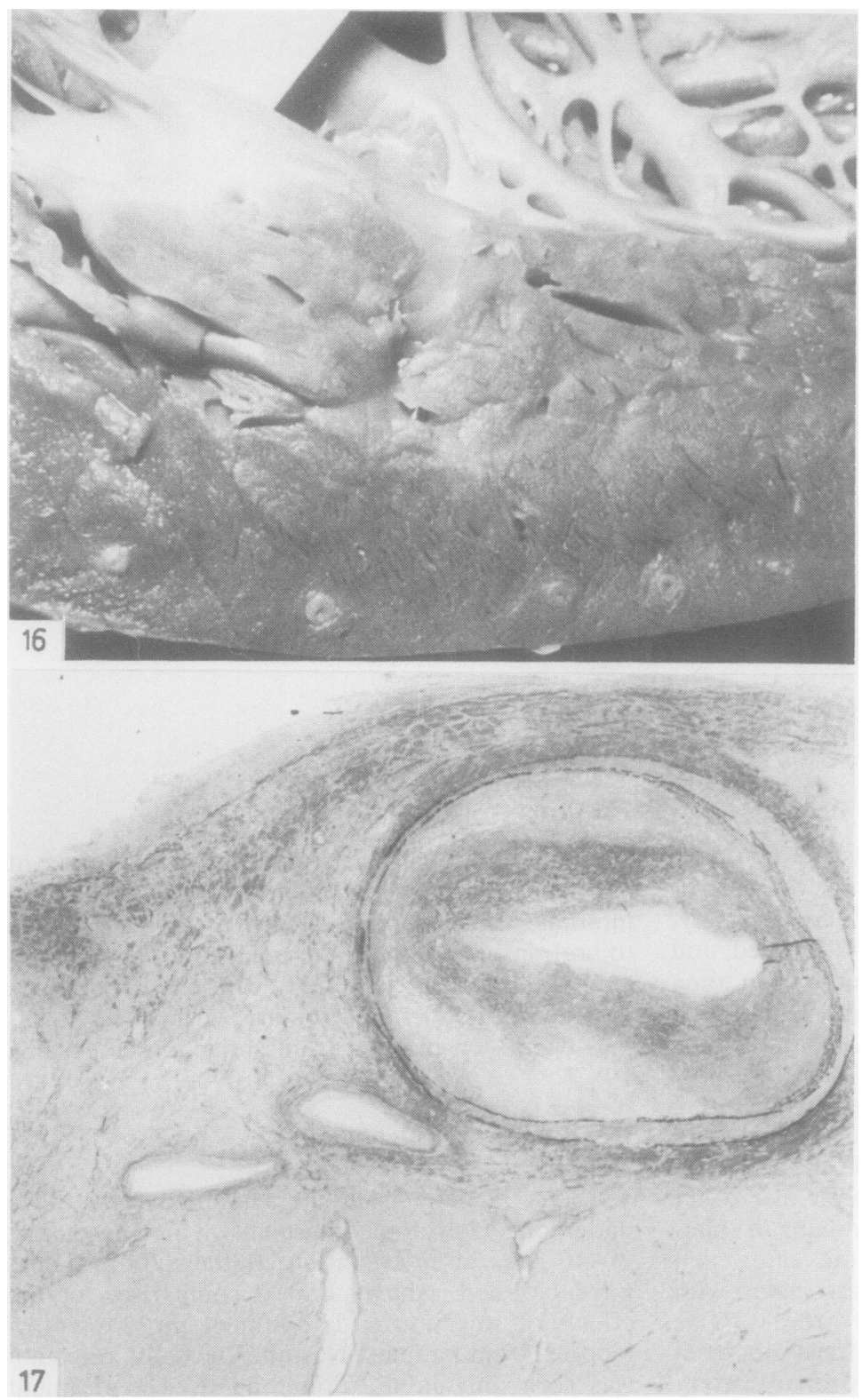

The findings in the three patients who died did not differ significantly from those in the orthotopic transplant deaths. Uys and Rose noted that both donor and recipient heart showed more severe changes than in orthotopic cases, but our three patients died between 12 and 46 days after operation, probably too early for advanced disease to develop.
Fig. 16 and 17 Coronary arteries in chronic rejection, taken from a patient who died suddenly 20 months after transplantation. Greatly thickened intramyocardial arteries can be seen on the cut surface of the left ventricle and the photomicrograph shows gross intimal thickening by fibrous and fibroblastic connective tissue with lipid areas. Photomicrograph, elastic van Gieson.

\section{Non-cardiac pathology}

The pathology of cardiac failure and therapeutic immunosuppression are well documented and will not be discussed here. Inevitably, all patients showed the former, and all who survived the first few postoperative days were liable to the latter.

The kidneys of patients receiving cyclosporin $\mathrm{A}$ 


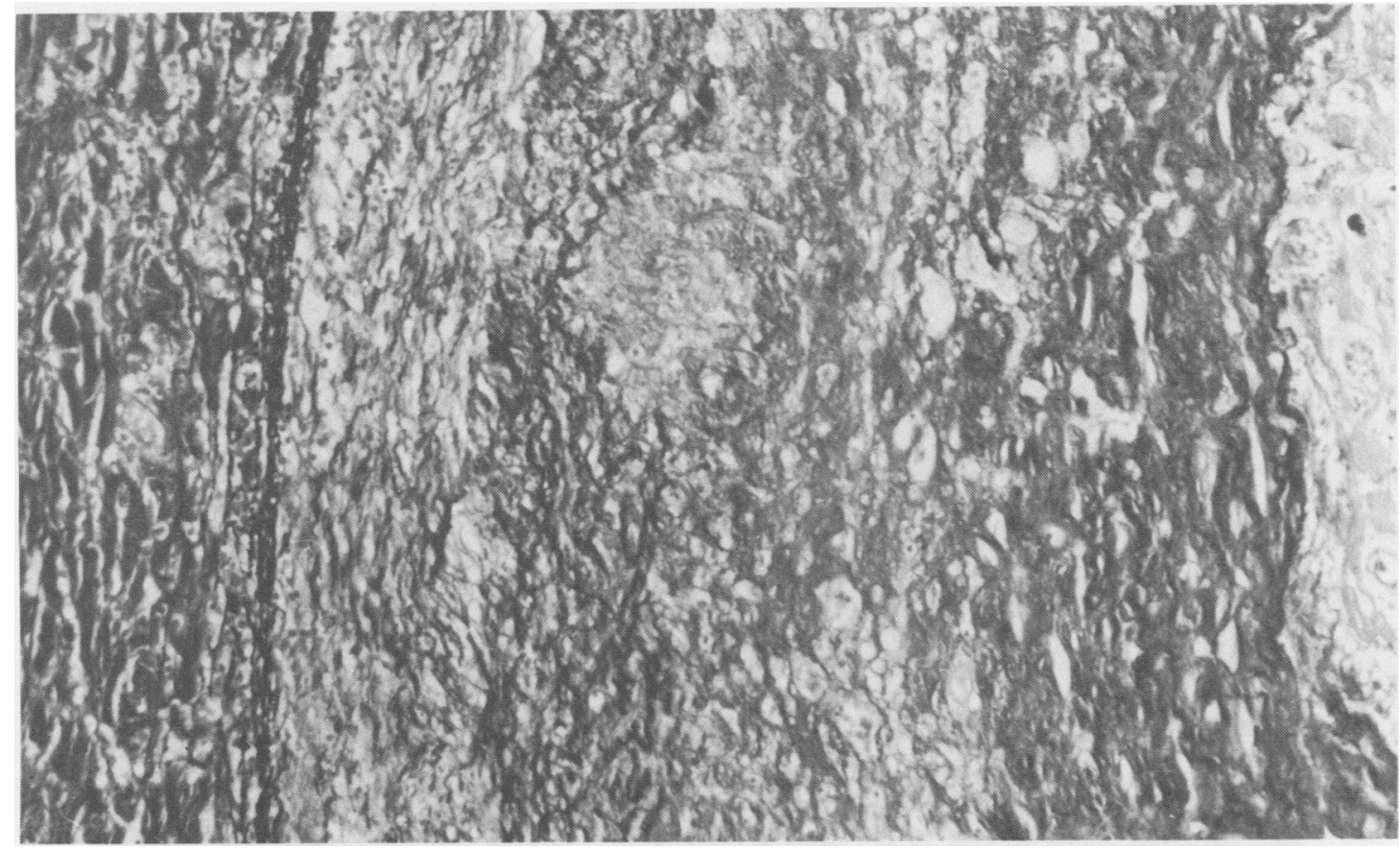

Fig. 18 High power view of part of a coronary artery showing foamy macrophages in fibrous thickened intima, with fibrin on the surface and fragmentation of medial elastic fibres. Elastic van Gieson.

are clearly of particular interest as these patients have impaired creatinine clearances and nearly all show at least moderate nephrotoxicity postoperatively. Most long term survivors require treatment for systemic hypertension. ${ }^{23}$ Apart from one patient with fatal renal failure our cases have simply shown changes consistent with past heart failure, terminal toxaemia, or autolysis. Our examination was limited to conventional histology on material taken at necropsy, however, and further study is clearly required to elucidate the cause of the altered renal function which seems to be specific to treatment with cyclosporin A.

\section{Neoplasia}

This final aspect of heart transplant pathology is not only a matter of concern for transplant recipients but is also clearly of interest in relation to aetiology of malignancy in general and lymphomas in particular. The subject has been reviewed by several authors. ${ }^{22-25}$ Some cases may be accelerated progression of a carcinoma that was already present but undetected, as occurred in one Papworth patient in whom an oat cell carcinoma became evident two months after transplantation; he died $\mathbf{4 4 1}$ days after the operation. Most, however, have been non-
Hodgkin's lymphomas, mainly large cell. ${ }^{2526}$ The three Harefield patients with malignant disease all had lymphoid neoplasms. One patient died of pneumonia 200 days after operation, and at necropsy a cerebral lymphoma of large cell type was found. The others developed a lung mass and mediastinal lymphadenopathy 10 months and two months, respectively, after operation and are both still alive. Lung or node biopsies in both cases showed typical non-Hodgkin's lymphoma morphology, monoclonal in the first case, and polyclonal in the second (Fig. 20).

Although an increased incidence of malignancy in transplant patients was recognised before the introduction of cyclosporin, the combination of cyclosporin with antithymocyte globulin or antilymphocyte globulin seems particularly linked with lymphoproliferative lesions. Our patients who developed lung and node lymphomas had been treated with this combination. The exact nature of the lymphoproliferative neoplasms is interesting and speculative. The histological features are those of lymphomas, but they may be mono or polyclonal; they appear to resolve if immunosuppression is reduced, but are invariably fatal otherwise. ${ }^{26}$ Immunosuppression was reduced in our patient with lung lymphoma and the lesion has resolved three months 


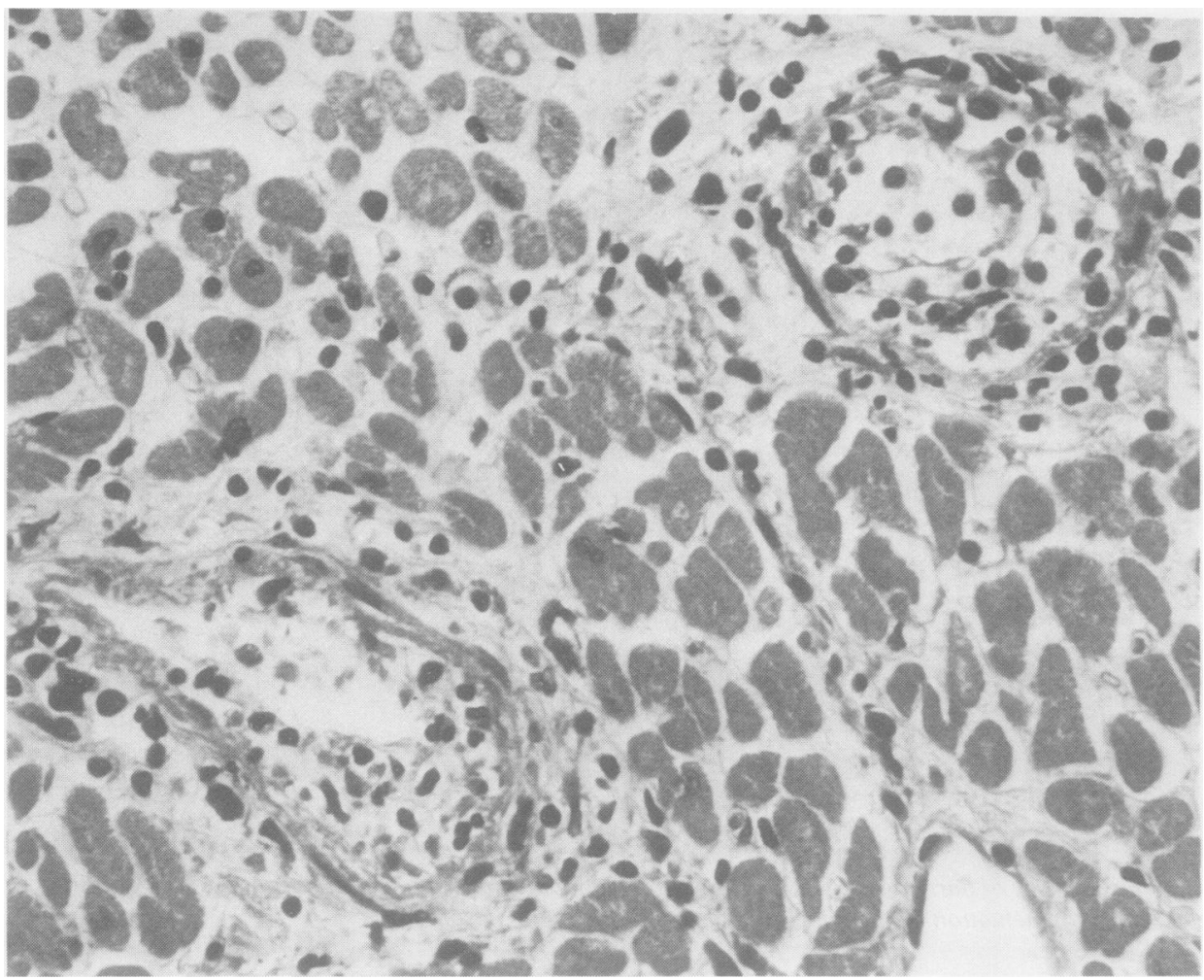

Fig. 19 Acute inflammatory changes in small intramyocardial coronary arteries in a patient who died suddenly 14 months after operation. Changes similar to those in Figs. 16 and 17 were also present. $H \& E$.

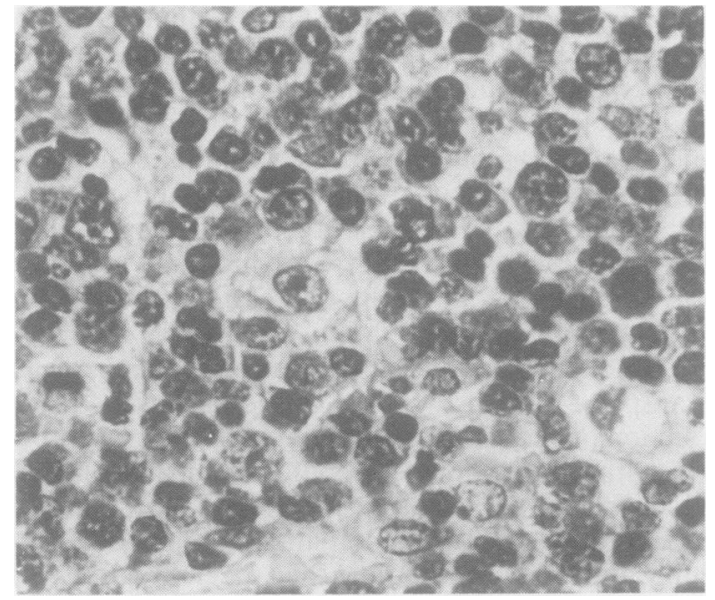

Fig. 20 Cyclosporin associated lymphoproliferative lesions presenting as mediastinal lymphadenopathy, about two months after transplantation. $H \& E$.

after diagnosis. The second patient's lymphoma also appears to be resolving but the reduced immunosuppression has been associated with further rejection episodes.
Thanks are due to the surgeons leading the two transplant teams, Terence English and Magdi Yacoub, and to the transplant officers, who provided much of the clinicopathological liaison, particularly Mr P Alivizatos. We also thank Mr L Beard, Papworth Hospital, and $\mathrm{Mr} \mathrm{R}$ Blake and $\mathrm{Dr} \mathrm{MH}$ Bennett, Mount Vernon Hospital, for help with the illustrations.

\section{References}

' Barnard CN. A human cardiac transplant: an interim report of a successful operation performed at Groote Schuur Hospital, Cape Town. S Afr Med J 1967;41:1257-74.

${ }^{2}$ Cooper DKC, Charles RG, Fraser RC, Beck W, Barnard CN. Long-term survival after orthotopic and heterotopic cardiac transplantation. Br Med J 1980;281:1093-6.

${ }^{3}$ Borel J-F. Immunological properties of cyclosporin A. Heart Transplantation 1982;1:237-42.

${ }^{4}$ Bieber CP, Griepp RB, Oyer PE, David LA, Stinson EB. Relationship of rabbit ATG serum clearance rate to circulating T-cell level, rejection onset and survival in cardiac transplantation. Transplantation Proceedings 1977;9:1031-6.

' English TAH, McGregor C, Wallwork J, Cory-Pearce R. Aspects of immunosuppression for cardiac transplantation. Heart Transplantation 1982;1:280-4.

- Devineni R, Keown N, McKenzie N, Stiller C, Kostuk WJ, Silver MD, Heimbecker RD. Cyclosporin in cardiac transplantation: 
Observations on immunologic monitoring, cardiac histology and cardiac function. Heart Transplantation 1983;2:219-23.

' Oyer PE, Stinson EB, Jamieson SW, et al. One year experience with cyclosporin $A$ in clinical heart transplantation. Heart Transplantaion 1982;1:285-9.

${ }^{8}$ Rabin BS. Immunologic aspects of human cardiac transplantation. Heart Transplantation 1983;2:188-91.

${ }^{`} \mathrm{O}$ 'Toole CM, Carvallo GS, Cranage MP, Large S. Immune responses in cardiac transplantation. 1. Detection of activated

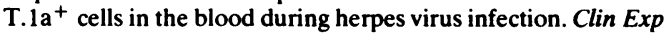
Immunol 1984;57:671-8.

${ }^{10}$ Caves PK, Stinson EB, Billingham ME, Rider AK, Shumway NE. Diagnosis of human cardiac allograft rejection by serial cardiac biopsy. J Thorac Cardiovas Surg 1973;66:461-6.

"Spiegelhalter DJ, Stovin PGI. An analysis of repeated biopsies following cardiac transplantation. Statistics in Medicine 1983;2:33-40.

${ }^{12}$ Billingham Margaret E. Diagnosis of cardiac rejection by endomyocardial biopsy. Heart Transplantation 1981;1:25-30.

${ }^{13}$ Montiès J-R, Goudard A, Avierinos C, et al. Thirteen years follow up of a heart transplanted patient. Heart Fransplantation 1982;1:128-37.

14 Billingham Margaret E. Progress in human pathology. Some recent advances in cardiac pathology. Hum Pathol 1979; 10:367-86.

is Cosimi AB, Colvin RB, Burton RC, et al. Use of monoclonal antibodies to $\mathrm{T}$-cell subsets for immunologic monitoring and treatment in recipients of renal allografts. $N$ Engl $J$ Med 1981;305:308-14.

${ }^{16}$ McGregor CGA, Fleck DG, Nagington J, Stovin PGI, CoryPearce R, English TAH. Disseminated toxoplasmosis in cardiac transplantation. J Clin Pathol 1984;37:74-7.
"Cebelin MS, Hirsch CS. Human stress cardiomyopathy. Myocardial lesions in victims of homicidal assaults without internal injuries. Hum Pathol 1980;11:123-31.

${ }^{18}$ McLeod AA, Neil-Dwyer G, Meyer CHA, Richardson PL, Cruickshank J, Bartlett J. Cardiac sequelae of acute head injury. Br Heart $J$ 1982; 47:221-6.

${ }^{14}$ Bieber CP, Stinson EB, Shumway N, Payne R, Kosek J. Cardiac transplantation in man. VII. Cardiac allograft pathology. Circulation 1970;41:753-72.

20 Jamieson SW, Oyer PE, Reitz BA, et al. Cardiac transplantation at Stamford. Heart Transplantation 1981;1:86-91.

21 Williams DM, Krick JA, Remington JS. Pulmonary infection in the compromised host. Am Rev Resp Dis 1976;114:359-94, 593-627.

${ }^{22}$ Uys CJ, Rose AG. The pathology of cardiac transplantation. In: Silver MD, ed. Cardiovascular pathology. Edinburgh: Churchill Livingstone, 1983;2:1329-52.

${ }^{23}$ Hunt SA. Complications of heart transplantation. Heart Transplantation 1983;3:70-4.

${ }^{24}$ Penn I. Problems of cancer in organ transplantation. Heart Transplantation 1982;2:71-7.

${ }^{25}$ Starzl TE, Porter KA, Iwatsuki S, et al. Reversibility of lymphomas and lymphoproliferative lesions developing under cyclosporin-steroid therapy. Lancet 1984;i:583-7.

20 Weintraub J, Warnke RA. Lymphoma in cardiac allotransplant recipients, clinical and histological features and immunological phenotype. Transplantation 1982;33:347-51.

Requests for reprints to: Dr PGI Stovin, Papworth Hospital, Papworth Everard, Cambs, England. 